\title{
Comunicación e integración en la era digital: un balance de la transición hacia la televisión digital en Brasil y Argentina**
}

pp. $29-50$

\section{HERnAN GALPERIN*}

En este trabajo se analizan las bases sobre las que se está desarrollando el proceso de transición hacia la TV digital en Brasil y Argentina, y se discuten las posibilidades que la transición presenta para replantear la estructura de mercado y el modelo de regulación de la TV abierta en los países del Mercosur. La principal hipótesis es que la Tv digital abre una oportunidad única para reformar el actual modelo de radiodifusión basado en la concesión de un número reducido de licencias a operadores de tipo generalista. Al multiplicar la capacidad de transmisión y facilitar el desarrollo de servicios interactivos tanto de entretenimiento como educativos y de información, la transición a la TV digital se ofrece como instrumento de política pública para alcanzar objetivos clave en materia de comunicación, como el pluralismo, la apertura del mercado y el achicamiento de la llamada brecha digital.
The basis, on which the transition process toward the digital Tv in Brazil and Argentina is developing, is analyzed in this article, and there is a discussion about the possibilities that the transition presents to reestablish the market structure and the regulation model of the open TV on the Mercosur countries. The main hypothesis says that the digital TV opens a unique opportunity to reform the broadcasting model operating today, which is based on a reduced concessions number of permissions given to operators of the generalist type. At increasing the transmission capacity and facilitating the development of interactive services, both those of entertainment and educative ones, the transition toward digital TV is offered as a public policy instrument to meet key targets related to communication, as pluralism, market opening, and slenderness of the so called digital gap.

\footnotetext{
*University of Southern California. email: hernang@usc.edu

**Trabajo presentado en el VI Congreso Latinoamericano de Investigadores de la Comunicación, junio 5-8, 2002, Santa Cruz de la Sierra, Bolivia.
} 
La televisión se encuentra ante el proceso de transición tecnológica más importante desde sus comienzos hace algo más de medio siglo. Este proceso implica profundos cambios en la organización industrial, el modelo de regulación y el papel socio-cultural del sector. La base de este proceso se encuentra en la digitalización de señales de televisión en las principales plataformas de transmisión: el cable, el satélite y la red terrestre de radiodifusión. La migración hacia la televisión digital hace posible la evolución de un modelo de radiodifusión, caracterizado por una limitada cantidad de canales, servicios unidireccionales y terminales de recepción de poca inteligencia, hacia un nuevo modelo caracterizado por una gran cantidad de canales, servicios interactivos y terminales inteligentes. Sin embargo esta migración, lejos de ser una simple transición tecnológica, exige una compleja coordinación entre los distintos sectores de la industria (fundamentalmente programadores, fabricantes de equipos receptores y operadores de redes), así como cuantiosas inversiones tanto de la industria como del público televidente. Además, al poner en cuestión las premisas básicas sobre las que se ha fundado el esquema de regulación analógico (limitada capacidad de transmisión, servicios unidireccionales, terminales "bobas", y una clara demarcación entre los servicios de radiodifusión y los de telecomunicaciones), la Tv digital abre una serie de interrogantes sobre cómo adecuar la reglamentación del sector a los nuevos parámetros político-económicos de la industria de radiodifusión.

Los principales socios del Mercosur se encuentran en pleno proceso de establecimiento de las bases sobre las cuales se dará la transición hacia la Tv digital, particularmente en lo que se refiere a los servicios de TV terrestre. Este proceso es de gran importancia en particular en los casos de Brasil y Paraguay, debido a que en estos países la gran mayoría de los hogares dependen de la recepción terrestre (Cuadro 1). Si bien el proceso de transición es aún incipiente en América Latina, es importante recordar que en toda evolución tecnológica existe un fuerte componente de dependencia del camino. ${ }^{1}$ Esto significa que pequeñas decisiones tomadas al inicio de la transición sobre normas tecnológicas y modelos de negocio tienden a delimitar la trayectoria futura de la industria, ya que con base en éstas los distintos actores sociales adaptan sus estrategias y efectúan inversiones de largo plazo. En otras palabras, estas decisiones de carácter técnico tomadas al inicio del proceso de transición van, poco a

${ }^{1}$ Sobre esta teoría y su aplicación en el análisis político, ver Zysman (1994). 


\section{CuAdro 1}

LAS TRES GENERACIONES DE LA TELEVISIÓN

Primera generación: Segundageneración: Tercerageneración: TV fordista TV posfordista TV digital

Servicios

$\begin{array}{lll}\text { Limitada cantidad de } & \text { Gran cantidad de } & \text { Servicios } \\ \text { servicios } & \text { servicios } & \text { personalizados e } \\ \text { unidireccionales de } & \text { unidireccionales de } & \text { interactivos de } \\ \text { radiodifusión } & \text { radiodifusión } & \text { radiodifusión y } \\ \text { masiva. } & \text { segmentada. } & \text { telecomunicaciones. }\end{array}$

Modelo de negocios

Publicidad masiva y/o Publicidad segmentada Publicidad subsidio guberna- y abonos. segmentada, abonos mental. y comisiones por transacción.

Estrategias de negocio

Derechos de propiedad Integración vertical Control de acceso y sobre el entre distribuidores y normas propietarias radioespectro. programadores. en el decodificador.

Modelo de regulación

Servicio público con Servicio privado con Por definirse. protección a los ciertas obligaciones concesionarios. públicas.

Fuente: propia.

poco, delimitando el modo de establecimiento de la nueva tecnología, y en definitiva demarcan la estructura futura del sector. Por ello, se hace importante discutir desde ahora las bases sobre las cuales los países del Mercosur encaran el proceso de reordenamiento de la industria de radiodifusión con base en la Tv digital.

En este trabajo se analizan los cambios en la estructura del mercado y en el modelo de regulación que se producen en los países desarrollados a partir de la transición a la TV digital, y se examinan las opciones que se abren a los países de América Latina en la realización de un plan de transición. La principal hipótesis es que la Tv digital abre una oportunidad única para reformar el actual modelo de radiodifusión basado en la concesión de un número reducido de licencias a operadores de tipo generalista. Al multiplicar la capacidad de transmisión de canales y hacer posible aplicaciones interactivas (tanto de entretenimiento como 
educativos y de información), la transición a la TV digital se ofrece como instrumento de política pública para alcanzar objetivos fundamentales en el área de las comunicaciones, como la diversidad de fuentes de información y el achicamiento de la llamada "brecha digital". Sin embargo, existen fuertes presiones políticas e inercias institucionales que hacen que, en muchos casos, se trate de adaptar la nueva tecnología al viejo modelo industrial y de regulación de la TV analógica. Es importante, pues, entender el abanico de oportunidades que ofrece la transición para democratizar el acceso a la infraestructura de comunicación, así como las fuerzas políticas e institucionales que, tanto en América Latina como en los países desarrollados, obstaculizan estos cambios.

En la primera parte del trabajo se discuten los desafíos de la TV digital al modelo de televisión presente, mientras que en la segunda se analiza la evolución de la transición en dos de los países que han liderado este proceso: el Reino Unido y EUA. En la tercera parte se discuten los pasos efectuados por Argentina y Brasil en la transición, con especial hincapié en el proceso de elección de la norma para TV digital en la región. Este proceso denota la falta de coordinación entre los socios del Mercosur al respecto (a diferencia, por ejemplo, de lo hecho por la Unión Europea), e ilustra las carencias institucionales del acuerdo en el sector de las comunicaciones. La conclusión del trabajo esboza una serie de propuestas para facilitar la colaboración entre los países del Mercosur en la integración de sus políticas de comunicación con el objetivo de avanzar en la formación de un bloque regional en este sector.

\section{LAS TRES GENERACIONES DE LA TELEVISIÓN}

En sus algo más de 50 años de historia, la televisión ha pasado por tres generaciones tecnológicas, cada una caracterizada por servicios, modelos de negocio y esquemas de regulación específicos (Cuadro 1). Es importante notar que cada nueva etapa no ha sustituido por completo a la anterior, sino que se ha agregado de forma gradual al modelo prexistente. Desde sus comienzos, hasta alrededor de los años setenta, los servicios de televisión consistían básicamente en un número limitado de canales terrestres de programación masiva financiados por publicidad (en euA y América Latina) y/o subsidios estatales (en la mayor parte de Europa y Asia). El modelo de regulación estaba basado, tanto para operadores privados como públicos, en la idea del "servicio públi- 
co": el Estado otorgaba un número limitado de concesiones para el uso del radioespectro a cambio de una serie de obligaciones formales respecto a la programación (programas educativos, de información, espacios de propaganda política, contenido nacional, etc.). El resultado fueron mercados de televisión oligopólicos, de programación poco diferenciada y, por lo general, altamente rentables para los pocos concesionarios privados. $\mathrm{La}$ justificación jurídica de este modelo descansa en la "escasez natural" del soporte de transmisión, o sea, el radioespectro. Sin embargo, numerosos estudios han demostrado que este justificativo no ha sido más que una estrategia de los distintos gobiernos por limitar artificialmente el número de concesiones, a fin de lograr ejercer mayor control sobre la programación, sea mediante concesiones directas a aliados políticos (típico en el caso de Brasil) o mediante un tácito intercambio de favores entre concesionarios privados y legisladores responsables por los otorgamientos y la eventual renovación de las licencias (el caso de EuA). ${ }^{2}$ En América Latina, este esquema garantizó por décadas una estrecha coalición entre un puñado de concesionarios privados y las élites cívico-militares, quienes cambiaban derechos cuasi monopólicos de radiodifusión por apoyo político-electoral. $^{3}$

Durante los años setenta una serie de cambios tecnológicos y regulatorios abrió las puertas al rápido desarrollo de la televisión por cable y, una década más tarde, al de servicios de satélite directo al hogar. Estos nuevos servicios de televisión multicanal permitieron ofrecer más (hoy día, mucho más) de lo mismo: programación enlatada en canales unidireccionales. Además, el desarrollo de tecnologías de control de acceso permite el desarrollo de un nuevo modelo de negocios basado en el abono por parte del usuario a distintos paquetes de programación, lo que ha hecho viables canales segmentados de audiencia reducida (de ahí el nombre posfordista). En el caso de la Tv multicanal, la principal estrategia de negocios es la integración vertical entre programadores y operadores de plataformas de transmisión, lo que permite ofrecer un paquete atractivo de contenidos para los abonados y el desarrollo de nuevos canales. El modelo de regulación de la Tv posfordista añade, al modelo de servicio público ciertos elementos de la llamada doctrina de las instalaciones esenciales (essential facilities), que otorga ciertos derechos de

\footnotetext{
$\overline{{ }^{2} \mathrm{El}}$ argumento de la escasez es además poco convincente, ya que es de esperar la escasez de un bien cuando el costo de adquisición es cero, y a la vez se lo puede comercializar en un mercado secundario.

${ }^{3}$ Entre la amplia literatura que documenta esta coalición, se destaca Fox (1990).
} 
acceso a la infraestructura de transmisión a programadores independientes y no comerciales (e.g., canales educativos y gubernamentales). Sin embargo, las plataformas de cable y satélite se han desarrollado esencialmente como redes cerradas, donde el operador de la infraestructura de transmisión ejerce un estrecho control sobre los contenidos y las aplicaciones desplegadas.

La tercera generación tecnológica comienza a principios de los noventa con el desarrollo de la transmisión digital de señales audiovisuales. A decir verdad, los principios técnicos de la Tv digital ya existían hace algún tiempo, pero lo que permite la realización comercial de los servicios es la caída significativa en los costos de los microprocesadores necesarios para codificación digital y posterior decodificación de las señales audiovisuales en tiempo real. La televisión digital consiste en el muestreo y codificación de las imágenes en un flujo de datos binarios (ceros y unos) que es transmitido a través de una red de transporte (terrestre, cable, satélite, etc.) hacia un aparato receptor que decodifica y reconstruye la imagen original. Este modo de transmisión presenta una serie de ventajas respecto a la transmisión analógica: 1) Mayor calidad de señales. La televisión digital posee mecanismos de corrección de errores que permiten mayor calidad de recepción (tanto de imágenes como de sonido) aun a igual nivel de resolución (número de líneas por cuadro) que en la televisión analógica.

2) Uso más eficiente del canal de transmisión. La compresión digital de señales permite utilizar el ancho de banda que hoy ocupa un canal analógico (6 mHz en América Latina) para transmitir entre cuatro y seis canales de baja resolución, o un canal de alta resolución (la llamada TV de alta definición, o TVAD) y otro de baja resolución. ${ }^{4}$ Además, la transmisión digital requiere una menor separación entre canales, lo que también optimiza el uso de radiofrecuencias.

3) Mayor flexibilidad en el uso del canal de transmisión. La técnica del multiplexing digital permite la asignación dinámica del ancho de banda de acuerdo con la necesidad de los distintos canales o servicios ofrecidos por el operador. ${ }^{5}$

$\overline{{ }^{4} \text { De }}$ hecho, la capacidad de un canal digital depende del sistema de modulación y de la red de transporte utilizada. El ejemplo dado corresponde a una red terrestre de transmisión.

${ }^{5}$ En la transmisión digital los programas con más cambios de imágenes o cámaras (por ejemplo, un partido de futbol) requieren un mayor flujo de información (y por lo tanto, mayor ancho de banda) que los programas más estáticos (por ejemplo, un 
4) Mayor interoperabilidad. El uso de la tecnología digital en la transmisión, almacenamiento y procesamiento de datos facilita la interoperabilidad con las aplicaciones y equipos de telecomunicaciones y la industria informática. Por ejemplo, los aparatos decodificadores de TV digital son esencialmente computadoras personales relativamente simples, lo que permite aprovechar el desarrollo y las economías de escala en ese sector.

En resumen, en el aspecto tecnológico la TV digital presenta una serie de ventajas en términos de calidad, cantidad y funcionalidad, tanto para los radiodifusores como para el público usuario, así como también para el gobierno en su papel de administrador del radioespectro y de promotor de espacios de difusión de información y entretenimiento. Sin embargo, la transición a la TV digital ha demostrado ser mucho más compleja de lo que muchos creían. En primer lugar, se requieren fuertes inversiones tanto de radiodifusores como de usuarios en la reconversión de los estudios, transmisores y el parque de receptores. ${ }^{6}$ Además, estas inversiones deben realizarse de manera coordinada en un contexto de alta incertidumbre sobre el modelo de negocios y el desarrollo futuro de la tecnología de TV digital. En segundo lugar, al alterar los parámetros económicos del sector, la TV digital presenta una serie de desafíos al modelo de regulación de la TV analógica. No se puede ya justificar la escasez de licencias terrestres en las limitaciones impuestas por el radioespectro. Además, se hace cada vez más difícil mantener la distinción entre servicios de radiodifusión y de telecomunicaciones, si bien éstos operan con base en modelos de regulación completamente diferentes. Por último, surgen nuevos problemas de competencia y estrategias de control del mercado, en particular referidos a las normas propietarias de acceso condicional y otras aplicaciones del decodificador, que exigen la adecuación del aparato jurídico a fin de que los entes reguladores puedan responder en forma adecuada. La siguiente sección analiza, precisamente, cómo han enfrentado estos desafíos económicos y jurídicos los países pioneros en TV digital: el Reino Unido y EuA.

programa de entrevistas). El multiplexing permite combinar las señales de distintos canales en un flujo único de datos.

${ }^{6}$ Se calcula que los radiodifusores deben invertir entre 500 mil y cinco millones de dólares, según la tecnología adoptada y la escala de la emisora, mientras que el público puede optar por invertir por encima de los dos mil dólares en un televisor digital, o bien hacerse de un decodificador cuyo precio gira sobre los 300 dólares (si bien, como veremos, éstos pueden estar subsidiados por las empresas o por el gobierno). 


\section{Dos MOdelos DE TRANSICIÓN: EL REINO UNIDO Y EUA}

Como vimos, uno de los pilares del modelo de regulación de la TV analógica ha sido el limitado número de concesiones de radiodifusión terrestre otorgadas por el Estado. Al reducir drásticamente el ancho de banda necesario para la transmisión de un canal, la Tv digital pone en cuestión la escasez del radioespectro y hace posible repensar por completo la lógica sobre la cual el Estado realiza las concesiones de televisión. Por supuesto, la posibilidad de abrir el mercado a nuevos competidores comerciales y a un sinnúmero de radiodifusores públicos y/o comunitarios es vista con poco agrado por los actuales beneficiarios del modelo analógico de regulación, es decir, los concesionarios de alta potencia, que por lo general ocupan los canales más deseables del radioespectro (la llamada banda VHF). Para ellos, la Tv digital es un arma de doble filo: por un lado, les permite explorar nuevos negocios y posicionarse mejor frente a la TV de paga y las nuevas plataformas de información y entretenimiento; por otro, exige altas inversiones en un mercado publicitario saturado y amenaza un cómodo oligopolio en el segmento terrestre. Una de las estrategias utilizadas por los actuales radiodifusores para promover la transición digital sin echar mano del mercado es plantear la necesidad de lanzar servicios de alta definición (TVAD). Al ocupar una mayor amplitud de banda por canal, la TVAD permite justificar la transición sin alterar la organización oligopólica del mercado. Si bien no está claro que la sociedad se beneficie al tener unos pocos canales digitales en alta definición en lugar de muchos canales digitales de baja definición, los concesionarios privados han hecho de la TVAD un mantra sin el cual, dicen, peligra la existencia misma de la radiodifusión gratuita.

El caso de EUA ejemplifica bien esta estrategia. Durante los años ochenta el organismo regulador, la Federal Communications Comission (FCC) recibió una serie de propuestas para reducir la cantidad de frecuencias dedicadas a servicios de televisión con el fin de hacerlas disponibles para telefonía móvil y otros servicios de telecomunicación. Frente a este asalto a lo que consideran un derecho de propiedad adquirido (si bien las propuestas no afectaban a ningún canal de televisión en funcionamiento), los radiodifusores decidieron plantear ante el Congreso estadunidense la necesidad de establecer servicios de TVAD. Como estos servicios requieren de un mayor ancho de banda (los prototipos existentes en la época, de origen japonés, requerían 8 MHZ ,es decir un 30\% más 
que los canales existentes), el argumento era que se debían reservar estas frecuencias para poner en marcha la transición a la TVAD. Cuando a comienzos de los años noventa una serie de desarrollos tecnológicos hicieron posible la transmisión digital de señales de Tv, la FCC optó inmediatamente por el desarrollo de una norma digital para la TVAD. Sin embargo, como vimos, esto abre un abanico de posibilidades que va más allá de los servicios de alta definición. Ya bloqueado el proyecto de reconvertir frecuencias de televisión para otros servicios, los radiodifusores argumentaron luego que, si bien la TVAD es deseable a largo plazo, en el corto plazo pretendían utilizar la TV digital para ofrecer más canales en baja resolución y nuevos servicios de transmisión de datos. En el Congreso, donde ya estaba avanzado un proyecto que otorgaba un segundo canal de 6 MHZ a las emisoras analógicas para ofrecer TVAD, varios legisladores plantearon que si el canal adicional no se iba a utilizar para servicios de alta definición, entonces porqué beneficiar exclusivamente a los radiodifusores ya existentes y no crear nuevas emisoras digitales tanto comerciales como públicas. Tras meses de debate sobre posibles licitaciones para los nuevos canales digitales, la Ley de Telecomunicaciones de 1996 finalmente convalidó el plan original y otorgó un segundo canal a todas las emisoras analógicas sin imponer obligación alguna respecto a transmisiones en alta definición. El valor del radioespectro otorgado sin costo alguno se estima en alrededor de 70 mil millones de dólares. ${ }^{7} \mathrm{Si}$ bien la ley dispone que las emisoras deben devolver los canales analógicos una vez concluido el periodo de transición a la TV digital en 2006 (para ser licitados a nuevos operadores de telecomunicaciones), en 1997 el Congreso decidió que la devolución se hará efectiva sólo cuando $85 \%$ de los hogares estén equipados para recibir TV digital. ${ }^{8}$ Dados los problemas que enfrenta la transición en EUA (que se discuten más adelante), se descarta que la devolución de los canales analógicos se haga efectiva en 2006. Según cálculos de los propios radiodifusores, bajo las reglas existentes este nivel de penetración se alcanzaría no antes de $2025 .{ }^{9}$

En el Reino Unido, por el contrario, el modelo de transición se ha basado no tanto en favorecer a los radiodifusores existentes sino en ampliar la oferta de programación y en crear mayor competencia en el mercado de la Tv de paga, dominado por el servicio satelital вskyв (par-

\footnotetext{
${ }^{7}$ Bazelon (1999).

${ }^{8}$ Balanced Budget Act of 1997.

${ }^{9}$ Ver Hazlett (2002).
} 
te del grupo multimedios News Corp.). En la Ley de Radiodifusión de 1996 el gobierno británico establece la disponibilidad a nivel nacional de seis frecuencias digitales para televisión. Éstas son otorgadas de la siguiente manera: la emisora pública BBC es la única que recibe una frecuencia digital entera con el fin de no sólo transmitir la programación existente en modo digital sino también de ampliar la oferta de canales públicos. ${ }^{10}$ Los demás radiodifusores analógicos (ITV, Channel 4, Channel 5, y la emisora pública de Gales $\mathrm{s} 4 \mathrm{C}$ ) deben repartirse otras dos frecuencias, mientras que las tres frecuencias restantes se ofrecen en licitación para un nuevo servicio de TV de paga. Este servicio, lanzado a finales de 1998, si bien enfrenta serios desafíos financieros, hoy ya supera el milón de abonados. El plan de transición adoptado en el Reino Unido se diferencia por su hincapié en ampliar la oferta de programación, incluyendo la pública, y por minimizar los costos de transición para los usuarios. Así, se han dejado para más adelante los servicios de TVAD, al requerir éstos mayor amplitud de banda y mayores costos de reequipamiento de receptores. El público se ha beneficiado por una oferta más variada de programación y la competencia entre los servicios de TV de paga, que ha obligado a los operadores a subsidiar en forma intensa el costo de los aparatos decodificadores. ${ }^{11}$ El éxito de este modelo se expresa claramente en la rápida adopción por parte de los usuarios británicos, que contrasta con la opaca recepción que ha tenido la TV digital en EuA (Cuadro 2).

Uno de los mayores problemas en EuA ha sido la falta de coordinación entre los distintos sectores involucrados en la transición a la TV digital, y la poca capacidad del gobierno para remediar esta falla en los mecanismos de coordinación del mercado. En toda transición tecnológica que involucra productos complementarios (en este caso, aparatos receptores, programación, transmisores, etc.) se requiere un cierto nivel de coordinación en términos de normas e introducción conjunta de productos al mercado. En caso contrario, obtenemos una típica paradoja del huevo y la gallina: el público no compra aparatos receptores de Tv digital porque la programación ofrecida es escasa y poco atractiva, los programadores no ofrecen más y mejores programas en formato digital porque existen pocos receptores, y los fabricantes venden pocos receptores, lo

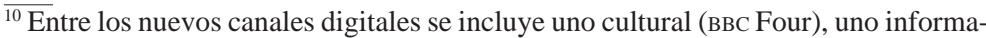
tivo (ввс News 24), y uno para niños (cBeebies).

${ }^{11}$ Tanto вskyв (TV digital satelital) como ITv Digital (nombre comercial del servicio de TV digital terrestre) ofrecen decodificadores gratis a nuevos abonados.
} 


\begin{tabular}{|c|c|c|}
\hline \multicolumn{3}{|c|}{$\begin{array}{c}\text { CUAdRo } 2 \\
\text { ESTABLECIMIENTO DE LA TV DIGITAL EN EUA Y EN EL REINO UNIDO }\end{array}$} \\
\hline & $E U A$ & Reino Unido \\
\hline Coordinación & $\begin{array}{l}\text { Librada al mercado, excepto } \\
\text { calendario de lanzamiento. }\end{array}$ & $\begin{array}{l}\text { Patrón europeo, gobierno } \\
\text { promueve activamente } \\
\text { transición. }\end{array}$ \\
\hline Costos & $\begin{array}{l}\text { Alto costo receptores para } \\
\text { usuarios. }\end{array}$ & $\begin{array}{c}\text { Costo receptores subsidiado } \\
\text { por operadores/gobierno. }\end{array}$ \\
\hline Licencias & $\begin{array}{l}\text { Radiodifusores analógicos } \\
\text { reciben canal adicional. }\end{array}$ & $\begin{array}{l}\text { Licitación para nuevos } \\
\text { operadores de multiplex } \\
\text { terrestres. }\end{array}$ \\
\hline $\begin{array}{l}\text { Modelo de } \\
\text { regulación }\end{array}$ & $\begin{array}{l}\text { Continuidad modelo TV } \\
\text { analógica. }\end{array}$ & $\begin{array}{l}\text { Separación de infraestructura } \\
\text { y contenido con base en } \\
\text { legislación telecomunica- } \\
\text { ciones. }\end{array}$ \\
\hline
\end{tabular}

Fuente: propia.

que los encarece (dada la falta de economías de escala), y lo que a su vez hace menos atractiva aún su compra por parte del público. Aunque hoy parece resuelta la batalla sobre la norma de transmisión en favor del sistema ATSC, ${ }^{12}$ quedan aún diversos aspectos por definirse, como la norma para conexión directa entre aparatos receptores digitales y los sistemas de cable, y la forma de proteger los derechos de propiedad intelectual sobre el contenido.

En el caso del Reino Unido, estos problemas de coordinación han sido minimizados dada la mayor capacidad de los gobiernos europeos para imponer normas sobre la industria, la mayor integración entre productores de contenido y operadores de servicios, y los objetivos menos ambiciosos de la transición. Luego del fracaso del llamado proyecto Eureka (respaldado por la Comunidad Europea, que buscaba imponer una norma de TVAD analógica llamada MAC), las autoridades europeas decidieron apoyar la norma de TV digital desarrollada por el grupo DVB (Digital Video Broadcasting), una asociación privada que agrupa a más de 300 radiodifusores, fabricantes, proveedores de TV de paga, entes reguladores y empresas de tecnología. Con el apoyo explícito de las autoridades comunitarias, este ente privado ha sido capaz de desarrollar un

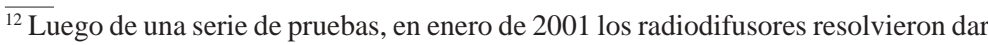
su apoyo definitivo al sistema ATSC, desarrollado por la llamada Grand Alliance y adoptado por la FCC en 1996 (Galperin 2002). 
sistema alternativo al ATSC, y además ofrecer credibilidad en lo que respecta a la adopción de la norma tanto a los usuarios como a los actores del mercado, sentando así las bases para el rápido crecimiento de los servicios de TV digital en el Reino Unido y otros países del viejo continente (ver Cuadro 2).

Por último, cabe destacar las diferencias en el modelo de regulación adoptado para la TV digital en ambos países. En el caso de EUA, la puesta en marcha de la TV digital terrestre se ha hecho con base en las reglas de juego heredadas de la TV analógica. Es decir, en cada mercado local se ha otorgado un número limitado de licencias a cambio de ciertas obligaciones formales en cuanto a la programación, que esencialmente sigue bajo el control de un puñado de concesionarios de escala nacional al igual que hace cinco décadas. En el Reino Unido, por el contrario, la puesta en marcha se ha efectuado con base en un modelo de separación entre aquellos que controlan la infraestructura de transmisión y ofrecen el servicio de televisión (los llamados operadores de multiplex), y aquellos que ofrecen la programación u otros servicios adicionales sobre esta infraestructura; en otras palabras, con base en un modelo más parecido al de la Tv por cable, lo que permite crear reglas tanto respecto al contenido como a la arquitectura de la red, a fin de limitar el control del mercado que ejercen los operadores de la red de transmisión. Las principales diferencias en los modelos de establecimiento de la TV digital entre EUA y el Reino Unido se resumen en el Cuadro 2.

\section{Los PRIMERos Pasos en AmÉrica Latina: los CASOS DE Argentina y BRASIL}

El proceso de transición a la TV digital en el Mercosur se inició a mediados de la década de los noventa, cuando las cámaras representantes del sector de radiodifusión y los gobiernos formaron grupos de trabajo con el objetivo de analizar la nueva tecnología y el modelo de adopción. El primer paso dado por estos grupos fue iniciar el debate sobre la normatividad para TV digital terrestre a ser adoptada en cada país. Como se ha discutido, el acuerdo de los distintos sectores involucrados en la transición (programadores, radiodifusores y fabricantes de equipos) alrededor de una norma única para TV digital resulta clave para crear coordinación y permitir inversiones a largo plazo en el reequipamiento del parque de transmisión y recepción. Actualmente compiten a nivel internacional tres normas distintas: el sistema estadunidense ATSC (Advanced 
Television System Committee), el sistema europeo desarrollado por el grupo DVB, y una variante más reciente de este último desarrollada en Japón por el grupo DiBEg (Digital Broadcasting Experts Group, que agrupa a las más importantes empresas electrónicas del país), llamado ISDB. Es importante notar que el desarrollo de tres sistemas (hasta ahora) de TV digital diferentes ha echado por tierra uno de los objetivos iniciales de la transición a la TV digital, que era justamente superar las barreras al comercio de equipos y programas presentadas por las distintas normas de televisión analógica de color adoptados en el mundo (basadas en tres sistemas primarios: el PAL, el NTSC y el SECAM). De hecho, a lo que asistimos hoy es a una repetición de la carrera neomercantilista de los años setenta, en la cual las empresas que controlan las patentes sobre estos distintos sistemas (ayer, de televisión a color; hoy, de Tv digital), apoyadas por sus respectivos gobiernos, presionan a los distintos países a adoptar sus sistemas. ${ }^{13}$

En Brasil, el proceso de elección comenzó en septiembre de 1994, cuando la SET (Sociedade de Engenharia de Televisão), que representa a los profesionales y empresas del sector y la ABERT (Associação Brasileira de Emissoras de Rádio e Televisão), que agrupa a los radiodifusores, formaron un grupo técnico para analizar la posible adopción de un sistema de TV digital. Este grupo técnico tendría luego gran importancia en el proceso de transición, al coordinar las pruebas técnicas que se realizan para elegir la norma. A partir de marzo de 1998, el nuevo ente regulador de las telecomunicaciones, Anatel (Agência Nacional de Telecomunicações) pasó a coordinar el proceso de selección. Es importante destacar que Anatel es responsable de la regulación del radioespectro, de los aspectos técnicos de la radiodifusión, y de las concesiones de TV de paga, pero no así de la concesión de licencias para radiodifusión terrestre, que continúa bajo las órbitas del Poder Ejecutivo (específicamente del Ministerio de Comunicaciones) y del Congreso. ${ }^{14}$

A fines de 1998 Anatel definió el plazo y las condiciones en que se llevarían a cabo las pruebas (de laboratorio y de campo) de los distintos sistemas de TV digital. Estas pruebas se realizaron entre octubre de 1999 y abril de 2000, coordinadas por el grupo de trabajo SET/ABERT. Además de las pruebas técnicas, Anatel aplicó entrevistas cualitativas y cuantita-

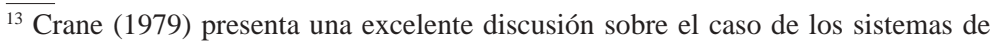
televisión de color. Sin embargo, en el caso de la TV digital es importante destacar que varias empresas tienen intereses en más de un sistema.

${ }^{14}$ Ver César Ramos (1997). 
tivas entre el público televidente. A mediados de 2000 se hizo público el resultado de las pruebas técnicas realizadas sobre los tres sistemas que, para sorpresa de muchos, revelaron una leve ventaja del sistema japonés ISDB, a pesar de que este sistema aún no ha sido implantado en la práctica en ningún país. Con estos resultados, en abril de 2001 Anatel inició una consulta pública para abrir el debate no sólo sobre los aspectos técnicos de los distintos sistemas sino también sobre su posible evolución tecnológica, el impacto sobre la industria nacional de equipos receptores y de transmisión, y la posibilidad de coordinar la elección de la normatividad con los países de la región. La consulta recibió más de 200 contribuciones de distintos actores económicos y sociales. Entre ellas se destacan las de los tenedores de las patentes de los tres sistemas en discusión, quienes además ejercieron una fuerte presión no sólo sobre Anatel sino también sobre el gobierno. A principios de 2002, cuando se esperaba ya una decisión final sobre la norma, Aanatel anunció la realización de una nueva consulta pública que abarcara no sólo los aspectos técnicos de la norma sino también los alcances económicos y sociales de la elección. Esta decisión reconoce que, más allá de los aspectos técnicos, la elección conlleva importantes implicaciones para el desarrollo tecnológico nacional y para el modelo de sociedad de información del país.

En el caso argentino, la iniciativa de la transición también concierne a las cámaras empresariales del sector, en especial a la ATA (Asociación de Teledifusoras Argentinas), que agrupa a los radiodifusores, y a CAPER (Cámara Argentina de Proveedores y Fabricantes de Equipos de Radiodifusión), que agrupa a los fabricantes de equipos. En julio de 1997 se creó, en el ámbito de la Secretaría de Comunicaciones, la Comisión de Estudio de Sistemas de Televisión Digital, cuyo objetivo es asesorar al gobierno en la elaboración de un plan de transición, incluyendo la elección de la normatividad más apropiada para el país. La Comisión quedó formada por representantes de las distintas cámaras empresariales, del Poder Ejecutivo y de los entes reguladores de televisión (el COMFER) y de las telecomunicaciones (la CNC). Un año más tarde el gobierno autorizó a las emisoras cabeceras de redes nacionales (asentadas en la ciudad de Buenos Aires) a realizar transmisiones experimentales de TV digital durante un plazo calculado en tres años. También se convocó a una audiencia pública sobre el modelo de transición y la elección de la normatividad. Sorpresivamente, y sin realizar prueba técnica alguna, en octubre de 1998 la Secretaría de Comunicaciones adoptó la Resolución 2357/98, que impuso el sistema estadunidense ATSC para la 
TV digital en Argentina. Según la Secretaría, la elección se fundamenta en la utilización de canales de 6 MHz en el país (similar ancho de banda que en EUA), debido a la necesidad de ofrecer servicios de TVAD (como vimos, no previstos inicialmente en el caso europeo), y a la pronta disponibilidad comercial de equipos de transmisión y recepción para la norma ATSC. Esta decisión generó fuertes cuestionamientos tanto dentro de la industria como por parte de asociaciones civiles. ${ }^{15} \mathrm{Al}$ cambiar el gobierno a fines de 1999, el nuevo secretario de Comunicaciones de la administración, De la Rúa, declaró que se pretendía revisar la decisión adoptada sobre TV digital, ya que la elección del sistema ATSC "se tomó sobre fundamentos incorrectos, con pruebas inexistentes, sin ningún tipo de coordinación con Brasil, y basándose en consideraciones falsas sobre la norma Europea DVB". ${ }^{16}$ A pesar de estas declaraciones, hasta la caída del gobierno de De la Rúa en diciembre de 2001, no se rechazó formalmente la Resolución 2357/98, por lo que sigue vigente la elección del sistema ATSC para Argentina. Sin embargo, es de esperar que la nueva administración del presidente Duhalde revise la medida una vez finalizado el proceso de selección en Brasil.

No cabe aquí evaluar las ventajas y desventajas de los distintos sistemas de TV digital, ya que se trata de un complejo tema de ingeniería de radiodifusión que va más allá del objetivo de este trabajo. Sin embargo, caben algunas reflexiones generales sobre el problema y sobre la manera en que se ha encarado la elección en Brasil y en Argentina. En primer lugar, si bien está claro que las pruebas técnicas pueden arrojar resultados significativos respecto a la adecuación de los distintos sistemas a las necesidades y geografía de los mercados nacionales, el desarrollo de la tecnología de TV digital depende en gran medida de las decisiones comerciales adoptadas y de la curva resultante de aprendizaje de los proveedores de la misma. Como lo ha demostrado Rosenberg (1994), cualquier norma técnica, una vez adoptada, tiende a perfeccionarse con el tiempo gracias a las inversiones en investigación y desarrollo y a la mayor experiencia de fabricantes e usuarios con la misma. En otras palabras, a largo plazo el sistema elegido tiende a perfeccionarse de modo tal que la elección parece haber sido la "correcta", cuando en realidad las otras también podrían haber resultado satisfactorias. Esto se evidencia por el desarrollo mismo que han tenido, en pocos años de utilización limitada, los distintos sistemas de TV digital. A modo de ejemplo, el sis-

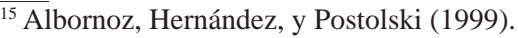

16 "Marcha atrás del país en norma para tv digital." Ámbito Financiero, 4/5/00.
} 
tema ATSC ha sido criticado por no haber sido diseñado para soportar aplicaciones móviles. Sin embargo, la cámara que representa el sistema ha manifestado a Anatel que ya se encuentran en desarrollo chips receptores avanzados que harían posible aplicaciones móviles sobre el sistema ATSC. ${ }^{17}$ Del mismo modo, mientras que el sistema DVв ha sido criticado por haber sido diseñado para canales de $8 \mathrm{MHz}$ (el ancho de banda utilizado en Europa para TV analógica) y por no soportar servicios de TVAD, en Australia ya se encuentran en funcionamiento servicios de TVAD con la norma DVB sobre canales de 7 MHz.

En definitiva, la elección de la normatividad para Tv digital trata menos de un problema técnico que de uno de política industrial para el sector de equipos de transmisión y recepción, así como de minimización de los costos de transición que deberán repartirse emisoras y usuarios. Cabe recordar que el desarrollo de las distintas normas para Tv analógica de color durante los años setenta, que provocó la fragmentación del mercado internacional de equipos y aparatos receptores, fue parte de una estrategia explícita de los gobiernos nacionales para fomentar la fabricación local de productos electrónicos y crear barreras noarancelarias a la programación extranjera. Desde la perspectiva del Mercosur, todos los sistemas de TV digital son de origen extranjero y, por lo tanto, los países del bloque deberán inevitablemente importar la tecnología (tanto en términos de componentes electrónicos como de pagos de regalías), y en cierta medida los mismos equipos de transmisión y recepción, al menos en la etapa inicial de la transición. En este contexto, el país que tiene más en juego es Brasil, ya que allí se concentra la mayor parte de la producción de aparatos receptores de la región (en la zona industrial de Manaos), de la cual se exportan más de un millón de unidades por año a países de América Latina, Europa y Asia. ${ }^{18}$ El gobierno brasileño ha reconocido la importancia de este aspecto en la decisión sobre la normatividad, así como el impacto de la transición en la balanza de pagos y en el desarrollo local de tecnología. ${ }^{19}$ De lo que se trata, como reconoce un estudio encargado por Anatel, es de elegir una norma que incentive la fabricación local de equipos receptores

${ }^{17}$ Ver "Comentarios Réplica del Advanced Television Systems Committee (ATSC)", Consulta Pública Anatel núm. 291, 23/7/01.

18 "Lucro de televisores está vindo do exterior". O Estado de São Paulo, 18/12/01.

${ }^{19}$ El grupo ABERT/SET ha calculado el costo total de la transición para la industria brasileña (cerca de ocho mil emisoras y repetidoras) en 1.700 millones de dólares. A esto debe sumarse el costo de reequipar el parque de 55 millones de aparatos receptores. 
y componentes, minimice los pagos en concepto de regalías, permita la participación regional en los foros internacionales de decisión sobre la evolución de la norma (ATSC, DVB, y DiBEG), y cree economías de escala para el intercambio regional de tecnología y de equipos..$^{20}$ En la adopción de la normatividad para Tv digital, la coordinación regional se hace entonces necesaria para mejorar la capacidad de negociación de los países frente al puñado de empresas que controlan la tecnología y el poder de inversión en la industria de equipos y componentes.

En segundo lugar, es interesante destacar las diferencias en el proceso político de elección del sistema de TV digital entre los principales socios del Mercosur. En el caso de Argentina, el proceso ha estado bajo la órbita directa del Poder Ejecutivo (por medio de la Secretaría de Comunicaciones), si bien se ha invitado a participar a representantes de los entes reguladores (el COMFER y la CNC) y de las cámaras industriales del sector. Como brazo administrativo del Ejecutivo, las decisiones de la Secretaría de Comunicaciones no están sujetas a los requerimientos de transparencia pública y justificación de decisiones, al que sí lo están las decisiones de un ente regulador independiente como Anatel. No sorprende pues que la apurada decisión de la Secretaría de imponer el sistema ATSC sin la realización de pruebas técnicas y luego de un efímero proceso de consulta pública, haya generado numerosas críticas entre radiodifusores, una protesta formal por parte de Brasil (por falta de cumplimiento de acuerdos de cooperación técnica en el marco del Mercosur), y hasta una presentación judicial en contra de la Secretaría. ${ }^{21}$ No sorprende tampoco que con el cambio de gobierno se haya dado marcha atrás a una decisión polémica tomada de forma prematura y poco transparente por el saliente gobierno de Ménem. En comparación, el largo proceso que viene liderando Anatel desde hace ya cuatro años, ha logrado sentar las bases de una elección que, si bien seguramente no conformará a todos, estará fundamentada en extensas pruebas técnicas y estudios, y conseguirá también una amplia participación de los sectores involucrados. Este proceso, al generar credibilidad respecto a la norma elegida, sienta bases firmes para que los actores del mercado y el gobierno emprendan las importantes inversiones de largo plazo que requiere la transición.

Ahora bien, mucho más importante que la elección de la normatividad es definir el modelo de transición a adoptar, cuyos aspec-

\footnotetext{
${ }^{20} \mathrm{CPqD}$ (2001).

21 “Marcha atrás del país en norma para TV digital.” Ámbito Financiero, 4/5/00.
} 
tos clave son el modo de otorgamiento de las nuevas licencias, el reparto de los costos de transición, el marco regulatorio para los nuevos servicios, y el periodo requerido para el cese de las transmisiones analógicas. El debate se ha centrado hasta ahora en el llamado modelo de negocio, es decir, sobre qué clase de servicios (TVAD, múltiples canales de TV en baja resolución, servicios móviles o de transmisión de datos) podrán ofrecer los futuros operadores de Tv digital. Sin embargo, es importante ampliar el debate a fin de trazar una estrategia de establecimiento de la TV digital que tenga en cuenta las necesidades del país en materia de infraestructura de información, así como los objetivos públicos en el área de contenidos. Como afirma César Ramos, el debate sobre el modelo de negocios de los operadores de TV digital debe ampliarse, al tratarse de "un conjunto de instituciones públicas y privadas que tienen enorme impacto sobre las sociedades contemporáneas en hechos políticos, económicos y culturales". ${ }^{22}$

Como se ha discutido, existen dos modelos básicos para la puesta en marcha: el modelo estadunidense que, al priorizar el otorgamiento de licencias a los radiodifusores existentes, supone cambios mínimos respecto al modelo de radiodifusión analógica, y el modelo británico, que supone mayores cambios al abrir el mercado a nuevos concesionarios y crear un marco regulatorio único para operadores de telecomunicaciones y televisión basado en los principios de redes abiertas y separación entre infraestructura y contenidos. Como es de esperar, tanto en Brasil como en Argentina los actuales concesionarios están ejerciendo fuertes presiones para que las licencias de TV digital se otorguen, en un principio, sólo a los canales analógicos, limitándose así la entrada de nuevos competidores con base en el modelo estadunidense. En otras palabras, si bien la transición a la TV digital, al reducir las limitaciones técnicas que impone la transmisión analógica, permite abrir el mercado de radiodifusión a nuevos competidores y expandir el alance de canales educativos y culturales, los actuales concesionarios pretenden minimizar el impacto de la transición sobre la estructura de mercado oligopólica heredada del modelo fordista de TV.

Lo interesante en el caso de Brasil es que, si bien no le corresponde a Anatel el otorgamiento de concesiones de radiodifusión, el ente regulador ya ha tomado medidas para reservar radiofrecuencias a fin de otorgar a cada emisora analógica existente, en 145 mercados locales, un

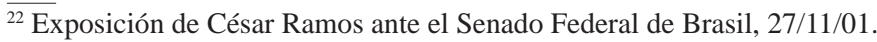


canal adicional para servicios de TV digital. ${ }^{23}$ Además, en una propuesta efectuada a Anatel, los actuales concesionarios sugieren que el canal adicional sea devuelto una vez que $90 \%$ de los domicilios en cada mercado adquieran un decodificador digital. ${ }^{24}$ Ahora bien, nuestro análisis de la experiencia estadunidense sugiere que, al no haber más incentivos que una mejor calidad de recepción (probablemente en la misma resolución, ya que los receptores de alta definición, aún fabricados en escala, tal vez se encuentren fuera del alcance de gran parte de la población brasileña), el público usuario tendrá pocos incentivos para adoptar la nueva tecnología. Esto podría llevar a décadas de duplicación en el uso de radiofrecuencias (es decir, que la misma programación se transmita en los sistemas analógico y digital), así como crear enormes obstáculos al lanzamiento de nuevas aplicaciones dependientes del uso de radiofrecuencias. Además, en este modelo los mismos licenciatarios analógicos que pregonan la transición tendrán pocos incentivos para estimular la adopción, ya que durante la etapa de transición gozarán de un canal adicional. Por último, adoptar este modelo significaría, como en el caso estadunidense, dar un paso atrás respecto a la posibilidad de separar el control sobre la infraestructura de transmisión, del control sobre los contenidos que abre el proceso de transición.

\section{ConClusión}

Los países del Mercosur, así como los de toda Latinoamérica, se hallan frente al reto que representa la llamada sociedad de la información. En un contexto en el que el desarrollo económico y cultural de los países depende cada vez más de su capacidad de producir y diseminar conocimiento de manera inclusiva, el debate sobre la evolución de una de las principales y más eficaces plataformas de transmisión de información —la televisión - adquiere una importancia que va más allá de mejorar la imagen en pantalla de las estrellas de telenovela o de los astros del futbol. Es importante recordar que, en contraste con la baja penetración de las computadoras personales y de la telefonía fija en América Latina, los receptores de televisión son ubicuos en el continente (Cuadro 3).

\footnotetext{
$\overline{{ }^{23}}$ Ver por ejemplo la resolución de Anatel sobre la planificación de radiofrecuencias para televisión (Plano Básico de Distribuiç,,o de Canais de Televisão em uHF e VHF, Resolução 291, 13/02/02).

${ }^{24}$ Comentarios del Grupo Técnico ABERT/SET a la Consulta Pública 291/2001 de Anatel sobre TV digital (disponible en www.anatel.gov.br).
} 


\begin{tabular}{|c|c|c|c|c|}
\hline Penetra & DE TECNOLOG & $\begin{array}{l}\text { CUADRO } 3 \\
\text { AS DE COMUN } \\
2000\end{array}$ & CACIÓN EN EL & MERCOSUR, \\
\hline & $\begin{array}{c}\text { TV abierta } \\
\text { (\% hogares) }\end{array}$ & $\begin{array}{l}T V \text { de paga } \\
\text { (\% hogares) }\end{array}$ & $\begin{array}{c}\text { Telefonía } \\
\text { (líneas c/100 } \\
\text { hab.) }\end{array}$ & $\begin{array}{c}\text { Usuarios } \\
\text { Internet } \\
(\% \text { hab. })\end{array}$ \\
\hline Argentina & 98 & 57 & 21 & 7 \\
\hline Brasil & 87 & 9 & 18 & 3 \\
\hline Uruguay & 98 & 47 & 28 & 11 \\
\hline Paraguay & 75 & 7 & 5 & 1 \\
\hline
\end{tabular}

Fuentes: ITU, OEA, Zenith Media.

Esto hace de la infraestructura de televisión terrestre una vía de acceso privilegiada para que la población del continente pueda acceder a las nuevas aplicaciones ligadas a la sociedad de la información, sean éstas educativas, informativas, o de entretenimiento. Vale recordar que aun en los países desarrollados, con mayor avance de la infraestructura de telecomunicaciones y menor disparidad en la distribución del ingreso, se reconoce este potencial. El gobierno británico, por ejemplo, ha declarado como prioridad que la TV digital posibilite el acceso a Internet a sectores de menores recursos económicos. ${ }^{25}$

Las decisiones sobre cómo llevar a cabo la nueva tecnología de radiodifusión digital son, por lo tanto, clave para definir cómo se repartirá el control de los flujos de conocimiento en la sociedad de la información en América Latina, ya que en la arquitectura técnica de la red se reparte el poder para decidir quién tendrá acceso a qué tipo de información y en qué condiciones. Como hemos visto, existen fuertes presiones sectoriales que favorecen la adaptación de la nueva tecnología al viejo modelo de la Tv analógica. Además, la inercia institucional tanto en la industria como en los entes reguladores, milita contra las propuestas de cambio. "La introducción de la Tv digital no puede, en ningún momento, causar ruptura en el statu quo vigente", ha declarado un alto funcionario de Anatel ante el Congreso brasileño. ${ }^{26}$ Pero es precisamente de esto que se trata: de aprovechar la oportunidad que ofrece la transición para replantear el modelo existente y alcanzar objetivos de larga data en mate-

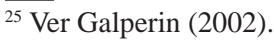

${ }^{26}$ Testimonio de Ara Apkar Minassian ante la Comisión de Ciencia, Tecnología, Comunicación e Informática de la Cámara de Diputados, 24/4/02.
} 


\begin{tabular}{lcc}
\hline \multicolumn{2}{c}{ CuAdro 4} \\
Sistema & EUA & \\
& & Peino Unido \\
Terrestre & 50,000 & \\
Cable & $11,100,000$ & $1,100,000$ \\
Satélite & $15,700,000$ & $1,500,000$ \\
Total hogares & $26,850,000$ & $5,300,000$ \\
Penetración total & $23 \%$ & $7,900,000$ \\
& & $32 \%$ \\
\hline
\end{tabular}

Fuentes: Digital tv Group, NCTA, Sky Report, New Media Markets, IDATE.

ria de comunicación, como el pluralismo, la democratización del acceso y la apertura del mercado a nuevos programadores públicos y privados. Al haberse debilitado las bases tecnológicas y políticas que daban sustento al viejo modelo de televisión, resta ahora forjar un nuevo modelo de radiodifusión terrestre basado en el derecho de acceso y en la arquitectura de redes abiertas. Cambiar un modelo institucional forjado hace casi cinco décadas no será fácil, pero la transición a la TV digital, al poner en cuestión las premisas del mismo, ofrece una interesante oportunidad.

\section{REFERENCIAS BIBLIOGRÁFICAS}

Albornoz, L., P. Hernández, y G. Postolski (1999) La televisión digital en la Argentina: Aproximaciones a un proceso incipiente, presentado en el XXII Congresso Brasileiro de Ciências da Comunicação, Rio de Janeiro, Brasil.

Bazelon, C. (1999) Completing the transition to digital television, Сво Papers. Washington, DC: Congressional Budget Office.

CÉsAr RAMos, M. (1997) "Uma nova legislação para as comunicaçôes brasileiras e o paradoxo da radiodifusão", Tendencias XXI, vol. 2, núm. 2, pp. 176-182.

CpqD (2001) Relatório integrador dos aspectos técnicos e mercadológicos da televisão digital. Disponible en www.anatel.gov.br.

CRANE, R. (1979) The politics of international standards: France and the color TV war. Norwood: Ablex.

Fox, E. (1990) Días de baile: El fracaso de la reforma de la televisión en Amé- 
rica Latina. México: Felafacs.

Galperin, H. (2002) "Can the us transition to digital Tv be fixed? Some lessons from two European Union cases", Telecommunications Policy, vol. 26, núms. 1 y 2 , pp. $3-15$.

Hazlett, T. (2002) “Abolish Television”, Financial Times, 6 de junio.

Office of Telecommunications (1998) Beyond the telephone, the television and the PC III. Londres: OFTEL.

Rosenberg, N. (1994) Exploring the black box. Nueva York: Cambridge University Press.

Zysman, J. (1994) "How institutions create historically rooted trajectories of growth", Industrial and corporate change, núm. 3, pp. 243-283. 